\title{
Suicide and non-suicide mortality after self-harm in Taipei City, Taiwan
}

\author{
Chian-Jue Kuo, David Gunnell, Chiao-Chicy Chen, Paul S. F. Yip and Ying-Yeh Chen
}

\section{Background}

Most previous studies of long-term mortality risk following self-harm have been conducted in Western countries with few studies from Asia.

\section{Aims}

To investigate suicide and non-suicide mortality after non-fatal self-harm in Taipei City, Taiwan.

\section{Method}

Prospective cohort study (median follow-up 3.3 years) of 7601 individuals presenting to hospital with self-harm (January 2004 to December 2006). Standardised mortality ratios (SMRS) for suicide and non-suicide mortality were calculated.

\section{Results}

Suicide risk in the year following self-harm was over 100 times higher than in the general population $(\mathrm{SMR}=119.6$, 95\% Cl 99.6-142.5). Males and middle-aged and older adults had the highest subsequent risk of suicide. Compared with people who took an overdose, individuals who used hanging or charcoal burning in their index episode had the highest risk of suicide. For non-suicide mortality the SMRs were 6.7 (95\% Cl 5.7-7.8) in the first year and 4.4 (95\% Cl 3.9-4.9) during the whole follow-up period.

\section{Conclusions}

Patterns of increased all-cause and suicide mortality following an episode of self-harm are similar in Taipei City to those seen in Western countries. Designing better aftercare following non-fatal self-harm, particularly for those with underlying physical disorders or who have used lethal self-harm methods, should be a priority for suicide prevention programmes in Asia.

\section{Declaration of interest}

None.
The risk of suicide among individuals who self-harm is considerably higher than in the general population. ${ }^{1-4}$ About half of individuals who attempt suicide have made unsuccessful attempts at least once before. ${ }^{1,5-7}$ Hence, people who self-harm are targeted in the suicide prevention strategies of many countries. ${ }^{8}$ Relatively few studies have explored mortality outcomes other than suicide among individuals who self-harm; although suicide is the main contributor to excess mortality, some studies have shown that other causes of death - particularly accidents, alcohol-related conditions, cancer, and respiratory, digestive and neurological diseases - are also increased considerably. ${ }^{3,9-11}$ Higher rates of concurrent physical illness, smoking and substance misuse are likely to contribute to this increased risk. ${ }^{3,9}$

Most previous follow-up studies of people who have selfharmed have been conducted in Europe, North America and Australia, ${ }^{4}$ with few studies from Asia. As the epidemiology of suicide varies in different cultures, studies conducted in the West may not be generalisable to Asia. One previous study of self-harm in rural Taiwan found an increased risk of suicide and non-suicide mortality in the first year following the index episode. ${ }^{12}$ However, because of the relatively small sample size $(n=1083)$, causespecific mortality could not be investigated. Furthermore, urban/rural differentials in suicide patterns are substantial in Asian countries, ${ }^{13-15}$ so this study's findings may not be applicable to patients seen in urban settings.

Using a representative sample of patients who had self-harmed presenting to services in Taipei City, this study investigates the short- and medium-term risk of suicide and non-suicide mortality. The study provides valuable information for suicide prevention programmes in Asia's emerging megacities.

\section{Method}

\section{Design, setting and study population}

We used a prospective cohort study to evaluate cause-specific mortality among residents of Taipei City, Taiwan. Taipei City, with a population of approximately 2.6 million, is the largest city in Taiwan. The city is relatively affluent - in 2009 over $99 \%$ of households owned a television and over $80 \%$ had a computer; per capita gross domestic product was US $\$ 48400$ and the literacy rate was over $99 \%{ }^{16}$ In 2010 the suicide rate in Taiwan as a whole was 16.8 per 100000 population and 12.6 per 100000 in Taipei City. The suicide figure in Taipei City was comparable to Hong Kong (13.8) but higher than in Singapore (8.0). ${ }^{17}$ The suicide rate in Taipei City is higher than many Western cities, for example suicide rates in London (UK) and New York City (USA) were 8.0 and 6.2 per 100000 population respectively in $2005 .^{18,19}$

Consecutive episodes of self-harm presenting to accident and emergency (A\&E) departments in Taipei City between January 2004 and December $2006(n=8343)$ were identified. Such cases are registered on the city's self-harm surveillance system - a suicide prevention programme run by Taipei City Suicide Research and Prevention Center. All hospital A\&E departments in the city are required to report cases of self-harm/suicide to the centre. These hospitals include 8 university-affiliated medical centres and 18 regional general hospitals. Cause-specific mortality was determined by linking the unique identification number for each person who self-harmed to official death records released by the Department of Health in Taiwan from 2004 to 2008.

\section{Study procedure}

Accident and emergency department staff are required to complete a structured case-note sheet for all self-harm presentations. The form records information on basic sociodemographic data and clinical characteristics of the individual as well as their treatment and management. These are then forwarded to Taipei City's Suicide Research and Prevention Center.

Self-harm is defined as an act of non-fatal intentional selfpoisoning or injury. For our current analysis, methods of self-harm were classified into solid/liquid poisoning, wrist cutting, charcoal burning, jumping from a height, hanging, 
self-mutilation (stabbing/slashing/cutting of body parts other than the wrists) and other methods. Solid/liquid poisoning was further categorised into medication overdose, pesticide poisoning and other poisons. If a person used more than one method of self-harm, we used a hierarchical algorithm based on method lethality as well as information derived from the case-note sheet to determine the key method of suicide. For example, if a person used hanging combined with medication overdose, then hanging was identified as the key method of suicide as it is a more lethal method than medication overdose. ${ }^{20}$ For those who used medication overdose together with wrist cutting or self-mutilation, cases were classified as wrist cutting or self-mutilation if the wound was potentially lethal and required immediate surgical suture; otherwise, medication overdose was identified as the key method for individuals who used these two methods simultaneously.

\section{Linkage with the national mortality database}

The unique national identity number was used to link the surveillance database with death records on Taiwan's national Death Certification System for deaths occurring between January 2004 and December 2008. The linkage process was approved by the Department of Health in Taipei City, and after it was completed, the national identity number of each participant was fully encrypted to preserve anonymity. The study was approved by the institutional review board of the Committee on Human Subjects of Taipei City Hospital, Taipei, Taiwan (ID: TCHIRB-991222-E).

The cause of death was classified according to ICD- $9^{21}$ recorded in the Death Certification System. For the present investigation, only the first reported self-harm episode was indexed. Individuals who died in the index episode $(n=202$, $2.4 \%$ ), were aged $<15$ years $(n=60,0.7 \%)$, not Taiwanese citizens $(n=98,1.2 \%)$ and those whose national identity number was not available $(n=382,4.6 \%)$ were excluded from the analysis. Our analysis is therefore based on a sample of 7601 individuals. Compared with the 7601 participants, those without available national identification number were more likely to be female $(n=298 / 382,78.0 \%$ v. $n=5296 / 7601,69.5 \% ; P<0.01)$ but did not differ in mean age $(36.2$ years $($ s.d. $=15.7)$ v. 35.7 years (s.d. =15.7); $P=0.53$ ) and method of self-harm (solid/liquid poisoning: $n=258 / 382,67.5 \%$ v. $n=5144 / 7601,67.7 \% ; P=0.96$; wrist cutting: $n=78 / 382,20.4 \% \quad$ v. $n=1502 / 7601, \quad 19.8 \%$; $P=0.75$ ); charcoal burning: $n=13 / 382,3.4 \%$ v. $n=356 / 7601$, $4.7 \% ; P=0.24)$.

Method of suicide was coded using ICD-9. Cases coded E952 were categorised as charcoal burning attempts, as previous research has shown that the majority of people attempting and dying by suicide by gassing in Taiwan use this method. ${ }^{22}$

\section{Statistical analysis}

We used the Kaplan-Meier method to display survival curves and estimate survival probability. Cox proportional hazards models were used to identify factors associated with the risks of suicide within 1 year as well as the risk during the total follow-up period. Relative risks were expressed as hazard ratios with 95\% confidence intervals, and covariates of the adjusted model include gender, age and the method used in the index episode of self-harm.

Standardised mortality ratios (SMRs) in the first year after the self-harm episode and during the whole follow-up period were calculated to compare the mortality of self-harm cases with that of the general population. In addition to suicide, all other top-ten categorised causes of deaths in Taiwan were assessed. We obtained rates of all-cause and cause-specific mortality for the general population to estimate SMRs from Taiwan's Department of Health Yearbook of Statistics. ${ }^{23}$ Relative to the general population with 5-year age bands for adjustment, SMRs were calculated by dividing the observed number of deaths by the expected number of deaths. ${ }^{24}$

\section{Results}

\section{Sample characteristics}

The rate of self-harm in Taipei City during the study period was estimated to be 154.6 per 100000 population (male rate: 108.5; female rate: 197.7). About two-thirds (69.5\%) of the cohort was female and more than half were aged less than 45 years. Median age for males and females was 34.0 years (interquartile range (IQR) 23.0-48.0) and 32.0 years (IQR 25.0-43.0) respectively. Solid and liquid poisoning was the most commonly used method (67.7\%).

After up to 5 years of follow-up (median 3.3 years, IQR 2.64.0 ), out of 7601 individuals, $201(2.6 \%)$ died by suicide and $315(4.1 \%)$ died from other causes. The total observation time was 24873.5 person-years and the suicide rate was 808 per 100000 per year.

\section{Standardised mortality ratios}

Gender-specific SMRs were estimated to compare mortality risk in the self-harm cohort with those of the general population (online Table DS1). Patients who self-harmed had a greater increased risk of all-cause mortality: SMR $=11.3$ (95\% CI 10.1-12.7) in the first year and 6.9 (95\% CI 6.3-7.5) over the entire follow-up period. The SMRs for each specific cause of death listed in Table 1 were elevated; the risk for suicide was highest $(\mathrm{SMR}=58.9)$, with the greatest risk $(\mathrm{SMR}=119.6)$ in the first year of follow-up. For non-suicide causes of death, accident mortality had the highest excess risk; SMRs were 12.8 and 7.9 in the first year and throughout the follow-up period respectively. When accidental deaths were subdivided into accidental poisonings and other types of accidents, the SMR for accidental poisoning (SMR =45.6) was much higher than for other types of accidental death $(S M R=4.7)$. The effect sizes of SMRs for death by accidental poisoning are similar to those for suicide, suggesting likely misclassification of some suicide deaths as accidents.

For other non-suicide and non-accidental causes of death, the SMR during the overall follow-up period ranged from 3.0 to 5.6. Risk of non-suicide mortality was higher among males than females (Fig. 1). Older adults had a much higher risk of non-suicide mortality compared with their younger counterparts (Fig. 1).

\section{Risk of suicide}

Approximately two-thirds of suicides ( $n=124,1.6 \%$ of patients who had self-harmed) occurred within a year of the self-harm episode (Table 1) and more than half ( $n=69,0.9 \%$ of patients who had self-harmed) of these deaths were within 3 months of the index episode. As the cohort was recruited over 3 years, this means that approximately 40 suicides per year in Taipei City occur among people who have recently (in the past year) attended hospital following self-harm. These deaths represent approximately $10 \%$ of Taipei's 420 suicides per year.

Furthermore, 46 males completed suicide within the first 3 months of the follow-up period, accounting for $50 \%$ of male suicide deaths, while relatively fewer females died by suicide within the first 3 month after self-harm $(n=23,21.1 \%$ of total 


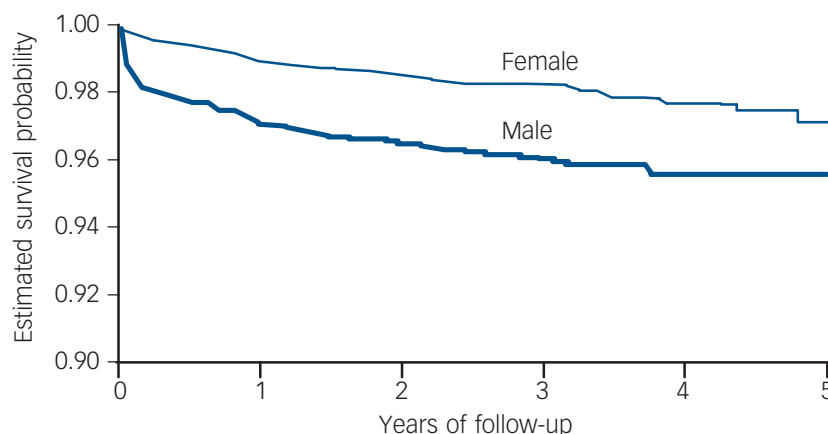

suicide mortality by gender

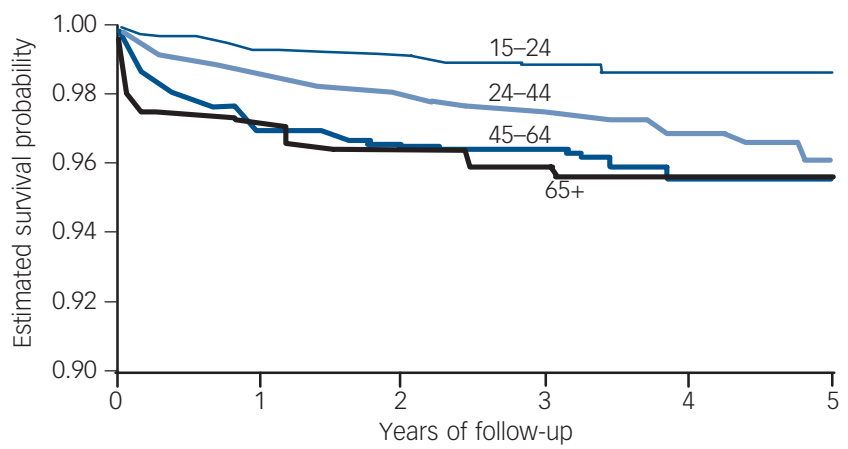

suicide mortality by age group

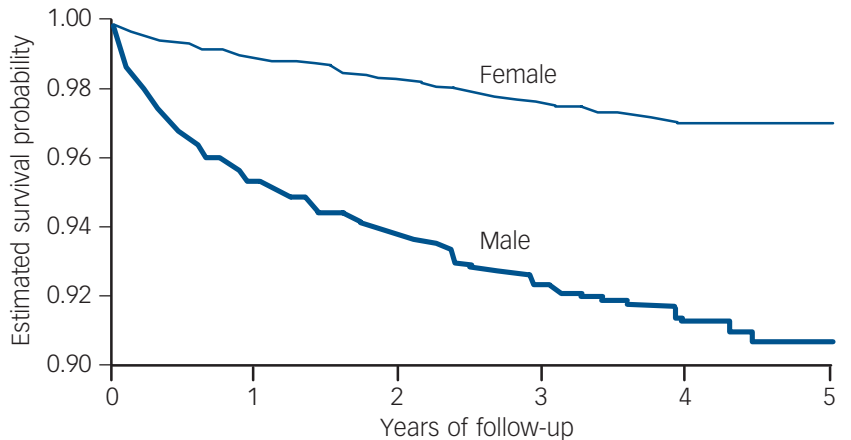

Non-suicide mortality by gender

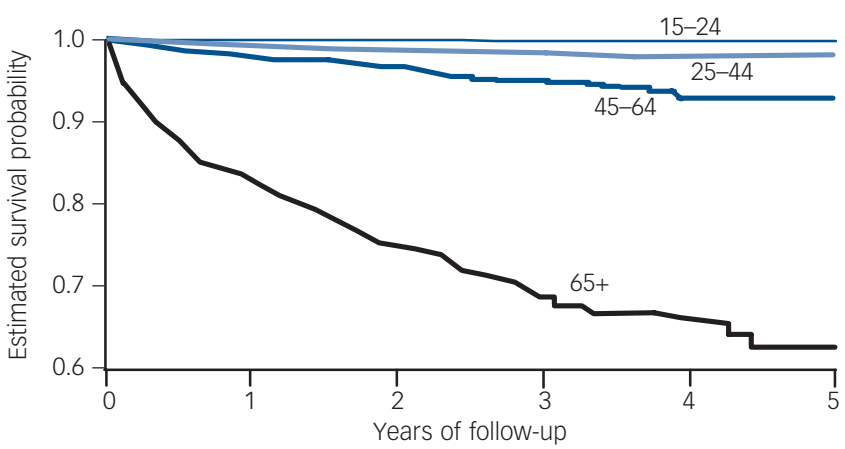

Non-suicide mortality by age group

Fig. 1 Estimated survival probability of subsequent mortality by gender and age group.

\begin{tabular}{|c|c|c|c|c|c|c|c|}
\hline \multirow[b]{2}{*}{ Characteristics } & \multirow[b]{2}{*}{$\begin{array}{c}\text { Number } \\
\text { of attempts }\end{array}$} & \multicolumn{3}{|c|}{ Suicide within 1 year $(n=124)$} & \multicolumn{3}{|c|}{ Suicide during follow-up $(n=201)$} \\
\hline & & $n(\%)$ & $\begin{array}{l}\text { Unadjusted HR } \\
\quad(95 \% \mathrm{Cl})\end{array}$ & $\begin{array}{l}\text { Adjusted } \mathrm{HR}^{\mathrm{a}} \\
\qquad(95 \% \mathrm{Cl})\end{array}$ & $n(\%)$ & $\begin{array}{l}\text { Unadjusted HR } \\
\qquad(95 \% \mathrm{Cl})\end{array}$ & $\begin{array}{l}\text { Adjusted } \mathrm{HR}^{\mathrm{a}} \\
\qquad(95 \% \mathrm{Cl})\end{array}$ \\
\hline \multicolumn{8}{|l|}{ Gender } \\
\hline Female & 5285 & $57(1.1)$ & 1.00 & 1.00 & $109(2.1)$ & 1.00 & 1.00 \\
\hline Male & 2316 & $67(2.9)$ & $2.8(1.9-3.9)^{\star * *}$ & $2.2(1.5-3.2)^{\star * *}$ & $92(4.0)$ & $2.0(1.5-2.7)^{\star * *}$ & $1.7(1.3-2.3)^{\star * *}$ \\
\hline \multicolumn{8}{|l|}{ Age, years ${ }^{b}$} \\
\hline $15-24$ & 1956 & $15(0.8)$ & 1.00 & 1.00 & 25 (1.3) & 1.00 & 1.00 \\
\hline $25-44$ & 3778 & $54(1.4)$ & $1.9(1.1-3.3)^{\star}$ & $1.9(1.0-3.3)^{*}$ & $105(2.8)$ & $2.2(1.5-3.5)^{\star \star *}$ & $2.2(1.4-3.5)^{\star * *}$ \\
\hline $45-64$ & 1321 & $40(3.0)$ & $4.0(2.2-7.3)^{\star \star *}$ & $3.4(1.8-6.2)^{* * *}$ & $51(3.9)$ & $3.2(2.0-5.2)^{\star \star *}$ & $2.9(1.8-4.7)^{\star * *}$ \\
\hline $65+$ & 528 & $15(2.8)$ & $4.1(2.0-8.4)^{\star * *}$ & $2.6(1.2-5.4)^{\star}$ & $20(3.8)$ & $3.6(2.0-6.5)^{* * *}$ & $2.6(1.4-4.8)^{\star *}$ \\
\hline \multirow{2}{*}{\multicolumn{8}{|c|}{$\begin{array}{l}\text { Index episode of self-harm } \\
\text { solid/liquid poisoning }\end{array}$}} \\
\hline & & & & & & & \\
\hline Medicine & 4720 & $56(1.2)$ & 1.00 & 1.00 & $98(2.1)$ & 1.00 & 1.00 \\
\hline Pesticide & 122 & $5(4.1)$ & $3.7(1.5-9.3)^{\star *}$ & $2.0(0.8-5.2)$ & $6(4.9)$ & $2.6(1.1-5.9)^{\star}$ & $1.7(0.7-4.0)$ \\
\hline Other poison & 302 & $8(2.6)$ & $2.3(1.1-4.8)^{*}$ & $2.0(0.9-4.1)$ & $12(4.0)$ & $2.0(1.1-3.6)^{*}$ & $1.8(1.0-3.3)$ \\
\hline Wrist cutting & 1502 & $15(1.0)$ & $0.8(0.5-1.5)$ & $1.0(0.6-1.8)$ & $29(1.9)$ & $0.9(0.6-1.4)$ & $1.1(0.7-1.7)$ \\
\hline Charcoal burning & 356 & $13(3.7)$ & $3.1(1.7-5.7)^{\star * *}$ & $2.4(1.3-4.4)^{\star *}$ & 19 (5.3) & $2.7(1.7-4.5)^{\star \star *}$ & $2.2(1.4-3.7)^{\star *}$ \\
\hline Jumping from a height & 87 & $1(1.1)$ & $1.0(0.1-7.1)$ & $0.9(0.1-6.4)$ & $1(1.1)$ & $0.6(0.1-4.0)$ & $0.5(0.1-3.9)$ \\
\hline Hanging & 77 & $12(15.6)$ & $15.8(8.5-29.5)^{\star \star *}$ & $10.3(5.4-19.8)^{\star * *}$ & $13(16.9)$ & $10.3(5.8-18.4)^{\star \star *}$ & $7.8(4.3-14.2)^{\star * *}$ \\
\hline Self-mutilation & 173 & $2(1.2)$ & $1.0(0.2-4.1)$ & $0.8(0.2-3.3)$ & $5(2.9)$ & $1.4(0.6-3.5)$ & $1.3(0.5-3.1)$ \\
\hline Other $^{d}$ & 260 & $12(4.6)$ & $4.0(2.1-7.5)^{* * *}$ & $2.7(1.5-5.2)^{\star *}$ & $18(6.9)$ & $3.5(2.1-5.8)^{\star * *}$ & $2.7(1.6-4.5)^{\star \star *}$ \\
\hline \multicolumn{8}{|c|}{$\begin{array}{l}{ }^{*} P<0.05 \text {; }{ }^{* *} P<0.01 ;{ }^{* * *} P<0.001 \text {. } \\
\text { a. Covariates of the adjusted model include gender, age, and the method used in index episode of self-harm. } \\
\text { b. Age not known for } 18 \text { individuals. } \\
\text { c. Index suicide attempt method not known for } 2 \text { individuals. } \\
\text { d. Other categories: domestic gas }(n=57) \text {, self-immolation }(n=9) \text {, tongue biting }(n=5) \text {, head banging }(n=25) \text {, neck slashing }(n=32) \text {, hit by a car }(n=4) \text {, drowning }(n=54) \text {, lying on the } \\
\text { rails }(n=17) \text {, motor vehicle exhaust gas }(n=18) \text {, jumping out of a car on a highway }(n=1) \text {, injecting unknown substances }(n=9) \text {, ingesting poisonous plants }(n=1) \text {, swallowing coins } \\
(n=3) \text {, electric shock }(n=1) \text {, using a firearm }(n=1) \text {, other unspecified methods }(n=23) \text {. }\end{array}$} \\
\hline
\end{tabular}


female suicides). Similarly, early risk was greater in those aged $>45$ years when compared with younger patients. A higher risk for suicide within the first year was detected among people who survived hanging (adjusted hazard ratio $(\mathrm{HR})=10.3$ ) and charcoal burning (adjusted HR=2.4) in their index episode (Table 1) compared with those who self-poisoned with solids and liquids. As for the risk of suicide throughout the whole follow-up period, the adjusted HRs were 7.8 for hanging and 2.2 for charcoal burning respectively. Although these two methods comprised only $5.7 \%(433 / 7601)$ of all self-harm cases, they accounted for $15.9 \%$ $(32 / 201)$ of all suicides because of their high lethality. After hanging $(\mathrm{HR}=15.8)$, pesticide poisoning was the method associated with the highest subsequent risk of suicide in unadjusted models $(\mathrm{HR}=3.7)$

The proportional hazards assumption was confirmed for all estimated variables in the first year of follow-up, but there was evidence of non-proportionality with respect to gender $(P=0.044)$ and hanging $(P=0.006)$ over the 5 -year follow-up period. Males and people who used hanging in the index episode were at relatively greater risk of suicide in the first year compared with subsequent years of follow-up.

\section{Methods used in index episode of self-harm and eventual suicide}

Only a quarter $(27.4 \%)$ of those who died by suicide used the same method as they adopted in their index episode of self-harm. However, those who used more lethal methods such as pesticide, hanging or charcoal burning tended to use the same method in both episodes (Table 2); $82 \%$ of individuals using these three methods used the same method in their subsequent suicide. Only $22(19.0 \%)$ of the suicides among those who self-poisoned used the same method to complete suicide, and self-harm by poisoning had one of the lowest incidence rates for eventual suicide.

\section{Discussion}

\section{Main findings}

We found that people who self-harm are at a greater increased risk of subsequent suicide, particularly in the first year after the index episode of self-harm. Furthermore, the risks of other non-suicide causes of death, particularly accidents, were also elevated. Both suicide and non-suicide mortality risks were particularly high among males. As expected, the highest mortality risk due to non-suicide causes was found among those aged >65 years; whereas for suicide, both middle (45-64 years) and older ( $>65$ years) age groups had higher risk when compared with younger people. Adopting hanging and charcoal burning at the index self-harm episode was associated with a markedly increased risk of later suicide completion; individuals who used these two lethal methods at the index self-harm episode were more likely to use the same method in subsequent completed suicide.

\section{Strengths and limitations}

To the best of our knowledge this is the largest study carried out in a non-Western country investigating mortality outcomes in a cohort of people who self-harm. Based on a city-wide self-harm surveillance system, selection bias is avoided. The strength of the study also lies in its large sample size; hence a wide range of causes of death can be assessed. However, several limitations should be recognised in the interpretation of the current findings. First, valid national identification numbers were not available for $4.6 \%$ of the cohort. Those who did not hold a valid identification card may be more likely to be individuals that are more disadvantaged in society (e.g. homeless, foreign brides); their risk of subsequent suicide may be even higher than estimated. However, as they comprised less than $5 \%$ of the cohort, our results would not be affected substantially. Second, the cohort included only people whose self-harm act led to an A\&E department visit. Individuals who had self-harmed but who did not visit A\&E were not included. In addition, some cases of self-harm may not have been registered with the suicide prevention centre: this cannot be ruled out. The incidence of self-harm among males was somewhat lower than that found in Western countries. According to a World Health Organization/EURO Multicentre Project on Parasuicide, the average male suicide attempt rate across European countries was 140 per 100000 population and the average female suicide attempt rate was 193 per 100000 population. ${ }^{25}$

In addition, detailed information on patient characteristics were not collected in the surveillance database, limiting our ability to control for some potential confounders or to make further analyses. For example, specific psychiatric diagnoses were not recorded and information on suicide intent or treatment were lacking.

\section{Elevated risk of suicide following self-harm}

In the first year after the index episode, $1.6 \%$ of patients who had self-harmed had killed themselves. After up to 5 years of follow-up, $2.6 \%$ of our sample had died by suicide - a 60 times greater risk than that of the general population. More than $60 \%$

Table 2 Method used in the index episode of self-harm and in the subsequent death by suicide among 201 people in Taipei City, 2004-2006, and followed up to 2008

\begin{tabular}{|c|c|c|c|c|}
\hline \multirow[b]{2}{*}{ Method } & \multicolumn{2}{|c|}{ Men } & \multicolumn{2}{|c|}{ Women } \\
\hline & $\begin{array}{l}\text { Index } \\
\text { attempt, } n\end{array}$ & $\begin{array}{c}\text { Same method at } \\
\text { eventual suicide, } n(\%)\end{array}$ & $\begin{array}{l}\text { Index } \\
\text { attempt, } n\end{array}$ & $\begin{array}{c}\text { Same method at } \\
\text { eventual suicide, } n(\%)\end{array}$ \\
\hline Solid/liquid poisoning & 48 & $10(20.8)$ & 68 & $12(17.6)$ \\
\hline Medicine & 36 & $5(13.9)$ & 62 & $9(14.5)$ \\
\hline Pesticide & 5 & $4(80.0)$ & 1 & $1(100.0)$ \\
\hline Other poison & 7 & $1(14.3)$ & 5 & $2(40.0)$ \\
\hline Wrist cutting & 11 & $0(0.0)$ & 18 & $0(0.0)$ \\
\hline Charcoal burning & 9 & $8(88.9)$ & 10 & $7(70.0)$ \\
\hline Jumping from a height & 0 & $0(0.0)$ & 1 & $0(0.0)$ \\
\hline Hanging & 10 & $8(80.0)$ & 3 & $3(100.0)$ \\
\hline Self-mutilation & 2 & $0(0.0)$ & 3 & $0(0.0)$ \\
\hline Other ${ }^{a}$ & 12 & $3(25.0)$ & 6 & $1(16.7)$ \\
\hline Total & 92 & 29 (31.5) & 109 & $23(21.1)$ \\
\hline
\end{tabular}


of the suicides occurred in the first year, approximately 120 times the risk when compared with the general population. Indeed, many prior studies have similarly reported a higher risk in the first year following self-harm. ${ }^{12,26,27}$ Our figure fell in the middle of an estimate from a systematic review, which reported that the risk of suicide in the first year of follow-up ranged between 0.5 and $2.0 \%{ }^{4}$ Our figure, however, is slightly lower than the $2.0 \%$ reported in a recent study from rural Taiwan. ${ }^{12}$ Although the age and gender distribution of the Taipei City sample was very similar to the sample from rural Taiwan, the suicide methods adopted were different. Individuals who self-harm in Taipei City more often take overdoses of medicine $(62 \% v .43 .7 \%)$ and few ingest pesticides $(1.6 \%$ v. $23.6 \%)$ compared with individuals in rural Taiwan. ${ }^{12}$ The differences may indicate different characteristics of self-harm populations in these two places; furthermore, pesticide ingestion is associated with a higher case fatality than medicine self-poisoning ${ }^{28}$ and so the higher relative use of pesticides in subsequent episodes in rural areas will contribute to higher subsequent suicide rates. A further explanation for the higher rate of suicide in rural Taiwan could be the lower availability and proximity of emergency treatment in rural areas, which could result in greater fatality.

In keeping with the majority of previous studies ${ }^{4,12,29-31}$ we found a higher risk of suicide among males who self-harm. In addition, half of the suicides among males occurred within the first 3 months after the index episode of self-harm, suggesting that intensive follow-up for males may be particularly important. However, it should be noted that the SMRs for suicide in males and females who self-harm were elevated to a similar extent at the end of follow-up. Many clinicians may perceive self-harm by females to be less serious than that in males. Our results, however, indicate considerable risk of suicide among females who self-harm.

Most studies have found that suicide risk increases with age among people who self-harm, ${ }^{11,12,29,32}$ although others have found no difference between age groups. ${ }^{31}$ As the majority (approximately $75 \%$ ) of people who self-harm are $<45$ years, large samples are needed to have sufficient power to detect any differences in the risk of suicide between age groups. With our large sample size, we were able to detect an increasing risk of suicide with increasing age. The risk was particularly high in the first 3 months of follow-up. Middle-aged people (45-64 years) have experienced the most dramatic increase in suicide rate in Taiwan. ${ }^{33}$ This underlines the need for clinicians to be particularly vigilant for suicide risk in older as well as middle-aged adults presenting with self-harm behaviours.

Similar to studies from Western countries, self-poisoning is the most common method of self-harm in our study sample. However, the proportion of people who used self-poisoning is somewhat lower in our participants $(68 \%)$, when compared with studies from the West. For example, the proportion of intentional self-poisoning among self-harm cohorts was reported to be $89 \%$ and $84 \%$ in the UK and Sweden respectively. ${ }^{29,34}$ A study from rural Taiwan reported the rate of self-poisoning to be $67 \%$; a figure similar to ours in Taipei City. ${ }^{12}$ Additionally, $5 \%$ of individuals who had self-harmed used charcoal burning - this may in part explain the lower rates of solid/liquid poisoning found in our cohort.

Use of hanging and charcoal burning for the index self-harm episode are associated with an increased risk of suicide during follow-up. In addition, the majority of those who subsequently died by hanging and charcoal burning used the same method in their index episode of self-harm. Our results echo a recent study from Sweden that concludes that individuals who use more lethal methods in non-fatal self-harm, including hanging, drowning, using a firearm or jumping from a height, have a greater risk of subsequent suicide. $^{34}$

In contrast to the findings from Sweden, ${ }^{34}$ we did not observe an increased risk of subsequent suicide after jumping from a height. It is possible that the majority of individuals choosing to jump from a height as a method of suicide in Taipei City are jumping from their own residential buildings, ${ }^{35}$ while in Sweden, jumping from a bridge is more common. Jumping from a bridge usually involves planning (e.g. transportation to jumping site) and hence may indicate a higher level of suicide intent, whereas jumping from a residential building may reflect an impulsive response rather than a strong intent to die. Furthermore, as most suicidal jumps are from high buildings in Taiwan, case fatality is likely to be higher than in Sweden, so the difference with the Swedish study may reflect the differences in characteristics of survivors from jumping suicide in these two places.

No information on suicide intent was available from our cohort. The relationship between the choice of method and suicide intent should be assessed in future studies to better inform the interpretation of case fatality associated with different methods. ${ }^{36}$ Our finding indicates that the method of self-harm should be taken into consideration in the assessment of people who self-harm.

Despite differences in patterns of suicide between Taipei City and Western cities, we found many similarities in the risk of suicide among our cohort. The similarities indicate that treatment guidelines or prevention strategies used in the West for individuals who seek medical help after self-harm may be equally applicable in Asia. However, the elevated risk of suicide among those who used charcoal burning in the index episode is worth noting, as charcoal burning is a novel and popular method of suicide in Asia. Current findings further highlight the importance of designing programmes that target this new method of suicide.

\section{Elevated risk of non-suicide mortality following self-harm}

An increased risk of mortality in self-harm populations is not due solely to raised rates of suicide. We found that patients who selfharm are at higher risk of mortality from a wide range of causes of death other than suicide. The overall non-suicide mortality risk was higher among men than women. Additionally, the risk was greater in the first year after the self-harm episode. Throughout the course of follow-up, we observed an excess of 4.4 times risk of dying from non-suicide causes when compared with the general population. The increased mortality risk covers a wide spectrum of diseases, including neoplasm, cardiovascular disease, diabetes mellitus, pneumonia, liver and kidney disease, and accidents. These conditions are themselves associated with an increased risk of suicide. ${ }^{37,38}$ Hence, a high proportion of self-harm patients may have pre-existing morbidity. The level of excess risk compared with the general population was higher than the risk observed in the $\mathrm{UK}^{10}$ and the USA, ${ }^{39}$ but lower than findings from Scandinavia. ${ }^{9,11}$ This difference probably reflects the differences in age distribution of self-harm samples or the variations of baseline disease rates between different countries.

There are several explanations for the excess risk of nonsuicide mortality in people who self-harm. First, the medical consequence of the self-harm incident may subsequently increase the risk of death. Second, certain physical disorders (e.g. disease of the gastrointestinal, cardiovascular, endocrine or immune system) may be related to psychiatric disorders ${ }^{40}$ which in turn increase the risk of suicide. Third, certain characteristics of individuals who self-harm such as impulsivity, risk-taking and alcohol misuse may increase the risk of death due to accidents or liver disease. 
Last, the presence of physical disease may in itself increase the risk of self-harm behaviours. ${ }^{37,38}$ This explanation is supported by our finding that the non-suicide mortality was extremely high among the elderly - a group that is likely to have a higher prevalence of physical morbidity.

Among all the non-suicide causes of death, the excess risk is highest for accidental death. Similar to suicide, the excess risk is particularly high in the first year after self-harm. In our current analysis, the SMR for accidental poisoning was much higher than other types of accident mortality and was similar to the suicide SMR, indicating the possibility of misclassification of suicide as accident mortality. This observation is consistent with a previous analysis of Taiwanese mortality data that indicated that many accidental poisoning deaths are likely to be misclassified suicides. ${ }^{41}$

\section{Clinical implications}

The timing of suicide following self-harm and the characteristics of those at greatest risk are broadly similar to those seen in previous studies from Western countries, indicating that strategies developed in the West to reduce suicide risk following self-harm may be applicable in Asia.

Individuals who self-harm are at an increased risk of premature death from suicide as well as other causes. This adds to the overall high risk status of these individuals. Hence, when dealing with patients who self-harm, clinicians should not only assess suicide risk, but also pay attention to their risk of other physical disorders. The first year after the self-harm incident is a critical period, as the risk of death is particularly elevated at this time. Mortality risk is higher for men and for those who are 45 years or over; the heightened suicide risk immediately after self-harm calls for more targeted efforts on this specific age and gender high-risk group.

Assessment of the method used should be a key element of suicide risk assessment. Our results clearly indicate that those who use highly lethal methods of suicide (i.e. hanging, charcoal burning, pesticide ingestion) are more likely to use the same method in any subsequent attempt. Future studies should explore further the reasons of method choice, their relationship with suicide intent and other psychiatric/physical factors to improve the positive predictive value of self-harm behaviours.

\section{Funding}

The study was supported by the Department of Health, Taipei City Government. The funding agency had no role in the design and conduct of the study; collection, management, analysis and interpretation of the data; and preparation, review or approval of the manuscript.

\section{Acknowledgements}

D.G. is an NIHR Senior Investigator. Y.-Y. C. takes responsibility for the integrity of the data and the accuracy of the data analysis, and that all authors had full access to all the data in the study.

Chian-Jue Kuo, MD, PhD, Taipei City Psychiatric Center, Taipei City Hospital, Taipei, and School of Medicine, Taipei Medical University, Taipei, Taiwan; David Gunnell, FFPH, DSC, School of Social and Community Medicine, University of Bristol, UK: Chiao-Chicy Chen, MD, PhD, Taipei City Psychiatric Center, Taipei City Hospital, Taipei, and School of Medicine, Taipei Medical University, Taipei, Taiwan; Paul S. F. Yip, PhD, Hong Kong Jockey Club Center for Suicide Research and Prevention, and Yip, PhD, Hong Kong Jockey Club Center for Suicide Research and Prevention, and
Department of Social Work and Social Administration, University of Hong Kong, Hong Kong; Ying-Yeh Chen, MD, SCD, Taipei City Psychiatric Center, Taipei City Hospital, Taipei, and Institute of Public Health and Department of Public Health, National Yang-Ming University, Taipei, Taiwan

Correspondence: Dr Ying-Yeh Chen, 309 Songde Road, Taipei City Psychiatric Center, XinYi District, Taipei, Taiwan. Email: ychen@tpech.gov.tw

First received 11 Jul 2011, final revision 1 Oct 2011, accepted 26 Oct 2011

\section{References}

1 Sakinofsky I. Repetiton of suicidal behaviour. In The International Handbook of Suicide and Attempted Suicide (eds K Hawton, K Van Heeringen): 385-404. John Wiley \& Sons, 2000.

2 Harris EC, Barraclough B. Suicide as an outcome for mental disorders. A meta-analysis. Br J Psychiatry 1997; 170: 205-28.

3 Hawton K, Fagg J. Suicide, and other causes of death, following attempted suicide. Br J Psychiatry 1988; 152: 359-66.

4 Owens D, Horrocks J, House A. Fatal and non-fatal repetition of self-harm. Systematic review. Br J Psychiatry 2002; 181: 193-9.

5 Appleby L, Dennehy JA, Thomas CS, Faragher EB, Lewis G. Aftercare and clinical characteristics of people with mental illness who commit suicide: a case-control study. Lancet 1999; 353: 1397-400.

6 Horton-Deutsch SL, Clark DC, Farran CJ. Chronic dyspnea and suicide in elderly men. Hosp Community Psychiatry 1992; 43: 1198-203.

7 Isometsa ET, Lonnqvist JK. Suicide attempts preceding completed suicide. Br J Psychiatry 1998; 173: 531-5.

8 Taylor SJ, Kingdom D, Jenkins R. How are nations trying to prevent suicide? An analysis of national suicide prevention strategies. Acta Psychiatr Scand 1997; 95: 457-63.

9 Ostamo A, Lonnqvist J. Excess mortality of suicide attempters. SoC Psychiatry Psychiatr Epidemiol 2001; 36: 29-35.

10 Hawton K, Harriss L, Zahl D. Deaths from all causes in a long-term follow-up study of 11,583 deliberate self-harm patients. Psychol Med 2006; 36: 397-405.

11 Nordentoft M, Breum L, Munck LK, Nordestgaard AG, Hunding A, Laursen Bjaeldager PA. High mortality by natural and unnatural causes: a 10 year follow up study of patients admitted to a poisoning treatment centre after suicide attempts. BMJ 1993; 306: 1637-41.

12 Chen VCH, Tan HKL, Chen C-Y, Chen THH, Liao L-R, Lee CTC, et al. Mortality and suicide after self-harm: community cohort study in Taiwan. Br J Psychiatry 2011; 198: 31-6.

13 Yip PSF, Callanan C, Yuen HP. Urban/rural and gender differentials in suicide rates: east and west. J Affect Disord 2000; 57: 99-106.

14 Chang SS, Sterne JA, Wheeler BW, Lu TH, Lin JJ, Gunnell D. Geography of suicide in Taiwan: spatial patterning and socioeconomic correlates. Health Place 2011; 17: 641-50.

15 Phillips MR, Li X, Zhang Y. Suicide rates in China, 1995-99. Lancet 2002; 359 : 835-40.

16 Nation Statistics, R.O.C. (Taiwan). Database. Nation Statistics, R.O.C. (Taiwan), 2011 (http://ebas1.ebas.gov.tw/pxweb/Dialog/ statfile1L.asp?lang=1\&strList=L).

17 Chen YY, Wu KCC, Yousuf S, Yip PSF. Suicide in Asia: opportunities and challenges. Epidemiol Rev (in press)

18 Mole G, Baker A. Suicide in London 2005-2007: An Update. Landon Health Observatory, 2009 (http://www.Iho.org.uk/viewResource.aspx?id=14779).

19 The Office of the Chief Medical Examiner of New York City. Vital statistics in 2005. New York State Department of Health, 2007 (http:// www.health.state.ny.us/nysdoh/vital_statistics/2005/table33a.htm).

20 Miller M, Azrael D, Hemenway D. The epidemiology of case fatality rates for suicide in the northeast. Ann Emerg Med 2004; 43: 723-30.

21 World Health Organization. Manual of the International Statistical Classification of Diseases, Injuries and Causes of Death (9th revision) (ICD-9). WHO, 1977.

22 Lin JJ, Chen LH, Huang SM, Lu TH. Problems in estimating the number of suicides by charcoal burning in Taiwan. J Epidemiol Community Health 2008; 62: 566 .

23 Department of Statistics. Yearbook of Statistics (2004-2008). Taiwan Department of Health.

24 Breslow NE, Day NE. Statistical methods in cancer research. Volume II - the design and analysis of cohort studies. IARC Sci Publ 1987; 82: 1-406.

25 Schmidtke A, Bille-Brahe U, DeLeo D, Kerkhof A, Bjerke T, Crepet P, et al. Attempted suicide in Europe: rates, trends and sociodemographic characteristics of suicide attempters during the period 1989-1992. Results of the WHO/EURO Multicentre Study on Parasuicide. Acta Psychiatr Scand 1996; 93: $327-38$

26 Yim PH, Yip PSF, Li RH, Dunn EL, Yeung WS, Miao YK. Suicide after discharge from psychiatric inpatient care: a case-control study in Hong Kong. Aust NZ J Psychiatry 2004; 38: 65-72.

27 Cooper J, Kapur N, Webb R, Lawlor M, Guthrie E, Mackway-Jones K, et al. Suicide after deliberate self-harm: a 4-year cohort study. Am J Psychiatry 2005; 162: 297-303 
28 Gunnell D, Eddleston M. Suicide by intentional ingestion of pesticides: a continuing tragedy in developing countries. Int J Epidemiol 2003; 32 902-9.

29 Hawton K, Zahl D, Weatherall R. Suicide following deliberate self-harm: long-term follow-up of patients who presented to a general hospital. Br J Psychiatry 2003; 182: 537-42.

30 Nordstrom $P$, Samuelsson M, Asberg M. Survival analysis of suicide risk after attempted suicide. Acta Psychiatr Scand 1995; 91: 336-40.

31 Suokas J, Suominen K, Isometsa E, Ostamo A, Lonnqvist J. Long-term risk factors for suicide mortality after attempted suicide: findings of a 14-year follow-up study. Acta Psychiatr Scand 2001; 104: 117-21.

32 Gibb SJ, Beautrais AL, Fergusson DM. Mortality and further suicidal behaviou after an index suicide attempt: a 10-year study. Aust NZ J Psychiatry 2005; 39: 95-100.

33 Law CK, Yip PSF, Chen YY. The economic and potential years of life lost from suicide in Taiwan, 1997-2007. Crisis 2011; 32: 152-9.

34 Runeson $B$, Tidemalm $D$, Dahlin $\mathrm{M}$, Lichtenstein $\mathrm{P}$, Langstrom $\mathrm{N}$. Method of attempted suicide as predictor of subsequent successful suicide: national long term cohort study. BMJ 2010; 341: C3222.
35 Chen YY, Gunnell D, Lu TH. Descriptive epidemiological study of sites of suicide jumps in Taipei, Taiwan. Inj Prev 2009; 15: 41-4.

36 Hawton K. Completed suicide after attempted suicide. BMJ 2010; 341: c3064.

37 Juurlink DN, Herrmann N, Szalai JP, Kopp A, Redelmeier DA. Medical illness and the risk of suicide in the elderly. Arch Intern Med 2004; 164: 1179-84.

38 Robson A, Scrutton F, Wilkinson L, MacLeod F. The risk of suicide in cancer patients: a review of the literature. Psychooncology 2010; 19: 1250-8.

39 Pederson AM, Tefft BM, Babigian HM. Risks of mortality of suicide attempters compared with psychiatric and general populations. Suicide 1975; 5: 145-57.

40 Sadock BJ, Sadock VA (eds). Psychological factors affecting medical condition and psychosomatic medicine. In Kaplan \& Sadock's Synopsis of Psychiatry: Behavioral Sciences/Clinical Psychiatry (9th edn): 822-50. Lippincott Williams \& Wilkins, 2003

41 Chang SS, Sterne JA, Lu TH, Gunnell D. 'Hidden' suicides amongst deaths certified as undetermined intent, accident by pesticide poisoning and accident by suffocation in Taiwan. Soc Psychiatry Psychiatr Epidemiol 2010; 45: $143-52$ 